\title{
Fine Sensorless Force Control using Diode-Clamped Linear Amplifiers
}

\author{
Yuki Yokokura*a) ${ }^{*}$ Member, Kiyoshi Ohishi* Senior Member
}

(Manuscript received July 1, 2013, revised Dec. 3, 2013)

\begin{abstract}
In this paper, diode-clamped linear amplifiers (DCLAs) are developed for achieving low-noise and fine force control. Class-B and Class-D power amplifiers are not suitable for driving a motor, owing to their power efficiency and high switching noise. In particular, a force control system using Class-D power amplifiers cannot generate appropriate fine force because of the switching operations by PWM or PDM. For that reason, we focus on DCLAs, which are capable of solving the problems both power efficiency and force noise. Furthermore, the force noise generated by the linear motor is less than that generated by the Class-D power amplifiers. Through a theoretical analysis, simulations, and experiments, the validity of the force control system using DCLAs is verified.
\end{abstract}

Keywords: force control, diode-clamped linear amplifier, motor drive, disturbance observer, motion control

\section{Introduction}

In the field of power electronics, primary target is reduction of power losses. To enhance the power efficiency, PWM (pulse width modulation) or PDM (pulse density modulation) inverters/converters utilizing switching devices have been much researched and developed ${ }^{(1)-(3)}$. In general, PWM inverters which consist of MOSFETs or IGBTs, are usually used in order to drive electric motors. Joule heat is minimized drastically, because the switching devices are operated in saturation mode or off state. Hence, the power efficiency of the inverters which is categorized as a Class-D power amplifier is very high. However, the typical Class-D power amplifiers generate the current noise as well as the desired current, due to PWM or PDM operations.

On the other hand, the field of motion control such as the velocity control ${ }^{(4)(5)}$, position control ${ }^{(6)-(9)}$ and force control ${ }^{(10)-(13)}$ mainly focuses on control performances of the electric motors. In case the noise occurs in the motor driver, the control performances, of course, deteriorate. In order to realize the fine motion control, low-noise Class-B power amplifiers can be utilized. The general Class-B power amplifiers are capable of generating the fine voltage and current waveform. From the view point of the performances of the motor drive, if possible the Class-B power amplifier should be utilized for advanced motion control such as the precise control $^{(14)}$, numerical controlled tools, high performance servo drive ${ }^{(15)}$, and real-world haptics ${ }^{(16)-(23)}$. However, the Class-B power amplifier cannot be used for driving high-power actuators because of the power efficiency.

In short, Class-B and Class-D amplifiers are not suitable for advanced motion control using high-power actuators.

a) Correspondence to: Yuki Yokokura. E-mail: yokokura@vos. nagaokaut.ac.jp

* Department of Electrical Engineering, Nagaoka University of Technology

1603-1, Kamitomiokamachi, Nagaoka, Niigata 940-2188, Japan

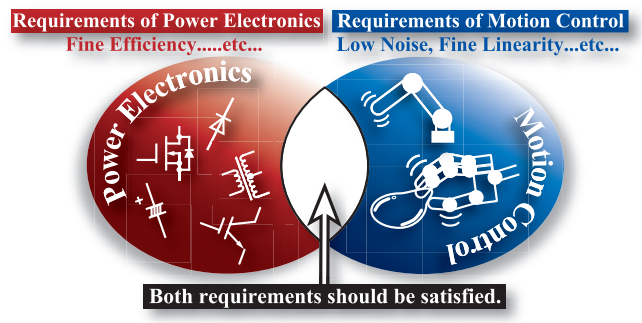

Fig. 1. Requirements of power electronics and motion control

Table 1. Advantages and disadvantages

\begin{tabular}{l|c|c|c}
\hline \hline Class of Amplifiers & Power Loss & Noise & Complexity \\
\hline Class-B & High & Low & Low \\
Class-D (PWM Inv.) & Low & High & Low \\
Diode-Clamped Linear Amplifier & Low & Low & High \\
\hline
\end{tabular}

The requirements both power electronics and motion control should be satisfied as shown in Fig. 1.

Meanwhile, Class-G power amplifiers are studied in the field of acoustoelectronics ${ }^{(24)-(27)}$. By using the Class-G power amplifiers, low-noise and fine power-efficiency operations are realized. In the field of motor drive, a Class-G amplifier for active magnetic bearings was used ${ }^{(28)}$. Moreover, Fujita et al. developed the diode-clamped linear amplifier (DCLA), which resembles to a multi-level inverter ${ }^{(29)(30)}$. In the case of DCLA, the respective MOSFETs are operated in linear state as well as saturation state or off state. Moreover, the difference from multi-level converters ${ }^{(31)}$ is that the p-channel MOSFETs are implemented. The linear amplifier proposed in Refs. (29), (30) carried out sinusoidal driving test of a induction motor to reduce harmonic current.

Table 1 summarizes the advantages and disadvantages of Class-B, Class-D (PWM inverter), and DCLA. DCLA has advantages from the viewpoint of the power loss and noise, although the complexity of the circuit becomes high.

In this paper, DCLAs for low-noise and fine force control 


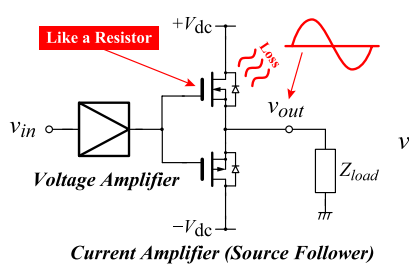

(a) Class-B power amplifier

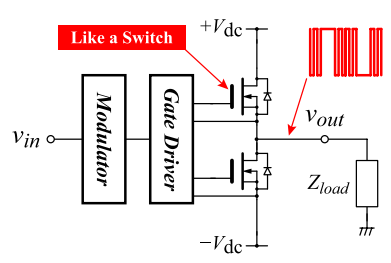

(b) Class-D power amplifier
Fig. 2. Circuit diagrams of power amplifiers

of a linear motor are developed. As mentioned above, the switching noise does not occur because DCLA is sort of a linear amplifier. Furthermore, the power efficiency of DCLA is almost equivalent to the Class-D power amplifier. Thus, the problems such as the power efficiency, the current noise, and the force noise are solved. This paper is organized as follows. In Sect. 2, classifications of the typical Class-B and Class-D power amplifiers are introduced. Topology, operations, power efficiency, and noise characteristics of DCLA are described in Sect. 3. In Sect. 4, the low-noise and fine force control of the linear motor with DCLA is explained. The numerical simulations and experiment of the force control system using the Class-D and DCLA power amplifiers are carried out in Sects. 5 and 6 Finally, this paper concludes the proposed approach relevant to the force control.

\section{Class-B and Class-D Power Amplifiers}

In this section, the classification of Class-B and Class-D power amplifiers is introduced.

2.1 Class-B Power Amplifier Figure 2(a) represents a circuit diagram of a general Class-B power amplifier, which consists of a voltage amplifier and MOSFETs. At first, the input voltage $v_{\text {in }}$ is amplified by the first stage voltage amplifier. The voltage signal is input to the gate pins of the MOSFETs. When the input voltage is positive, the low side MOSFET is off state, and the high side MOSFET is in active operations. Thus, the high side MOSFET is equivalent to sort of a resistor, which consumes the power given by

$$
P_{D}=V_{D S} I_{D}
$$

where $P_{D}, V_{D S}$, and $I_{D}$ denote the power loss of the MOSFET, the voltage between the drain and the source, and the drain current. In general, the power efficiency of the Class-B push-pull amplifier $\eta_{b}$ is derived as

$$
\eta_{b}=\frac{\pi}{4}=0.785
$$

on condition that phase factor is equal to $1^{(29)}$.

Since the Class-B amplifier is basically categorized as a linear amplifier, the noise of output voltage and current is reduced. Compared with a Class-D amplifier mentioned as below, the power loss of the Class-B amplifier is high. Hence, the Class-B amplifier is not suitable for driving high-power actuators from view point of energy-saving.

2.2 Class-D Power Amplifier Figure 2(b) depicts a circuit diagram of a general Class-D power amplifier. As with the Class-B amplifier, the two MOSFETs are used in order to drive the load. However, the MOSFETs are operated in a saturation mode, so that the MOSFETs are on-state or off-state depending on the gate signal. According to (1),

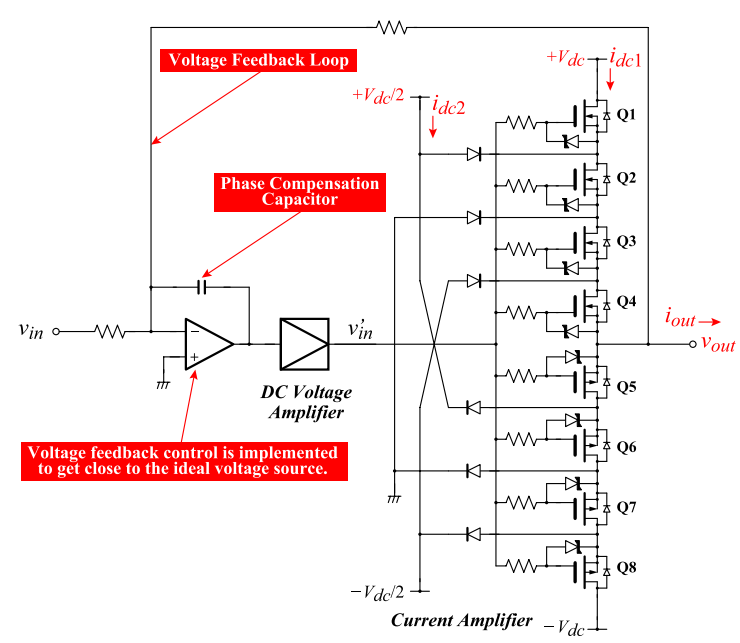

Fig. 3. 4-series DCLA with voltage feedback

Table 2. Operations of MOSFETs in 4-series DCLA

\begin{tabular}{c|c|c|c|c|c|c|c|c}
\hline \hline Range of input voltage $v_{i n}^{\prime}$ & Q1 & Q2 & Q3 & Q4 & Q5 & Q6 & Q7 & Q8 \\
\hline $0<v_{i n}^{\prime}<\frac{V_{d c}}{2}$ & OFF & Active & ON & ON & ON & Active & OFF & OFF \\
$v_{i n}^{\prime}=\frac{V_{d c}}{2}$ & OFF & ON & ON & ON & ON & OFF & OFF & OFF \\
$\frac{V_{d c}}{2}<v_{i n}^{\prime}<V_{d c}$ & Active & ON & ON & ON & Active & OFF & OFF & OFF \\
$v_{i n}^{\prime}=v_{d c}$ & ON & ON & ON & ON & OFF & OFF & OFF & OFF \\
\hline
\end{tabular}

Table 3. Power efficiency vs. number of series

\begin{tabular}{l|c|c|c|c|c|c}
\hline \hline Num. of series $\ell$ & 4 & 6 & 8 & 10 & 12 & 14 \\
\hline Num. of MOSFETs & 8 & 12 & 16 & 20 & 24 & 28 \\
Efficiency $\eta_{D C L A}[\%]$ & 84.2 & 87.7 & 89.9 & 91.4 & 92.5 & 93.4 \\
\hline
\end{tabular}

the power losses of the MOSFETs are theoretically zero, because the either $V_{D S}$ or $I_{D}$ equals to zero in a steady state. In general, the power efficiency of the Class-D amplifier is approximately over $90 \%$. Therefore, PWM or PDM inverters which is categorized as the Class-D amplifier, have been much used for driving high-power motors.

However, the gate signal is modulated by PWM or PDM in order to generate arbitrary output voltage. The modulated pulse is filtered typically by the load $Z_{\text {load }}$ and/or filters. However, the output current of the Class-D amplifier still includes the noise caused by the modulation. For this reason, the Class-D amplifier is not suitable for advanced motion control.

\section{Diode-Clamped Linear Amplifier}

3.1 Topology and Operations Figure 3 shows a circuit diagram of a DCLA. DCLA consists of MOSFETs, gate resisters, diodes, and Zener diodes. As with the Class-B amplifier, the high side MOSFETs should be chosen as a n-type MOSFET, and the low side MOSFETs should be selected as a p-type MOSFET.

In order to prevent breakdown of a gate of the MOSFETs, Zener diodes are connected between the gate pins and the source pins. $V_{d c}$ represents the dc link voltage. The dc link power supplies are connected to the each drain pins through the Schottky diodes. Fundamentally, DCLA is operated as a source follower. Therefore, the voltage amplification is almost 1 . Hence, it is necessary to implement the voltage amplifier in front of the leg.

Even where the forward admittance of the MOSFETs is large value, the output voltage $v_{\text {out }}$ decreases at heavy load. 


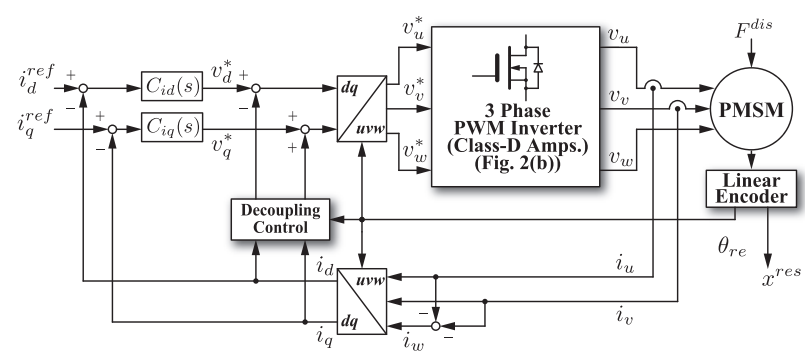

Fig. 4. Block diagram of current control system with Class-D power amplifiers

The cross over distortions also occur. In Ref. (32), the analog voltage feedback control using operational amplifiers has been implemented. In this paper, we also utilize the operational amplifier for realizing the output voltage control. The output voltage is fed back to a operational amplifier as shown in Fig. 3, to get close to the ideal voltage source. When oscillations and/or overshoot are caused, the capacitor should be connected as shown in Fig. 3, to stabilize the voltage feedback. The operations of MOSFETs in a DCLA is shown in Table 2.

3.2 Power Efficiency The theoretical power efficiency of a DCLA $\eta_{D C L A}$ is derived as Ref. (29)

$$
\eta_{D C L A}=\frac{\pi \ell^{2}}{16 \sum_{k=1}^{\frac{\ell}{2}} \sqrt{\ell k-k^{2}}}
$$

where $\ell$ denotes the number of series. Table 3 represents a relation between the power efficiency and the number of series. According to (3), the number of series should be set as large from view point of the power efficiency.

3.3 Noise Characteristics As mentioned in Sect. 2, the voltage of the Class-D amplifier is modulated by PWM or PDM in order to generate the desired voltage. On the other hand, PWM or PDM are unused in the case of DCLA. Therefore, the voltage and current noise cased by the switching operations of PWM or PDM does not occur.

\section{Low-Noise Force Control}

In this section, the force control system with inner current controllers is explained. At first, this paper describes the current controllers using a general PWM inverter, which consists of three Class-D power amplifiers. This paper discusses about the theoretical current noise of a Class-D amplifier. The current control system with DCLA and the sensorless force control are mentioned in Sects. 4.3 and 4.4.

4.1 Current Control using Class-D Power Amplifiers

Figure 4 shows a block diagram of the current control system with the Class-D power amplifiers, which drive the linear motor. The current responses of phase $\mathrm{u}, \mathrm{v}$, and $\mathrm{w} i_{u}, i_{v}, i_{w}$ are transformed into the current responses on d-q coordinate $i_{d}$, $i_{q}$ according to the electric angle $\theta_{r e}$. The voltage references on d-q coordinate $v_{d}^{*}, v_{q}^{*}$ are calculated as

$$
\left[\begin{array}{c}
v_{d}^{*} \\
v_{q}^{*}
\end{array}\right]=\operatorname{diag}\left(C_{i d}(s), C_{i q}(s)\right)\left(\left[\begin{array}{c}
i_{d}^{r e f} \\
i_{q}^{r e f}
\end{array}\right]-\boldsymbol{T}_{\theta_{r e}}\left[\begin{array}{c}
i_{u} \\
i_{v} \\
i_{w}
\end{array}\right]\right)
$$

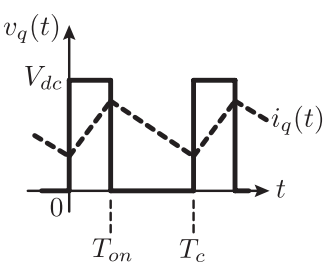

(a) PWM voltage and current

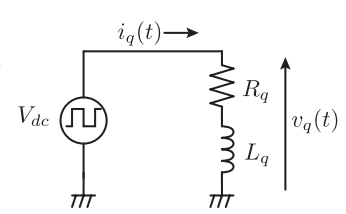

(b) R-L circuit
Fig. 5. Electrical systems driven by Class-D amplifiers

where $i_{d}^{r e f}$ and $i_{q}^{r e f}$ denote the current references on d-axis and q-axis, respectively. $i_{d}^{\text {ref }}$ is set to 0 , because field weakening is unused. $\boldsymbol{T}_{\theta_{\text {re }}}$ represents a transformation matrix for Clark and Park transform. The linear motor used in this paper is SPMSM(surface permanent magnet synchronous motor). $C_{i d}(s)$ and $C_{i q}(s)$ denote current controllers, which is expressed as

$$
C_{i d}(s)=C_{i q}(s)=K_{i p}+\frac{K_{i i}}{s}
$$

In (5), the each gain $K_{i p}$ and $K_{i i}$ are designed as

$$
\begin{aligned}
& K_{i p}=\omega_{c} L_{q} \\
& K_{i i}=\omega_{c} R_{q}
\end{aligned}
$$

where $L_{q}$ and $R_{q}$ represent the inductance and resistance of the motor on q-axis. $\omega_{c}$ denotes the control bandwidth of the current controllers.

In order to reduce the affect of interferences between $d$ axis and q-axis, a decoupling controller is implemented. The voltage references on $\mathrm{d}-\mathrm{q}$ coordinates are transformed into the 3-phase voltage references $v_{u}^{*}, v_{v}^{*}, v_{w}^{*}$ as follows:

$$
\begin{array}{r}
{\left[\begin{array}{c}
v_{u}^{*} \\
v_{v}^{*} \\
v_{w}^{*}
\end{array}\right]=\boldsymbol{T}_{\theta_{r e}}^{T}\left(\left[\begin{array}{c}
v_{d}^{*} \\
v_{q}^{*}
\end{array}\right]+\dot{\theta}_{r e} L_{d q}\left(\boldsymbol{I}_{2}-\mathbf{1}\right)\left[\begin{array}{c}
i_{d} \\
i_{q}
\end{array}\right]+\left[\begin{array}{c}
0 \\
e_{q}
\end{array}\right]\right)} \\
\ldots \ldots \ldots \ldots \ldots \ldots \ldots \ldots \ldots \ldots \ldots \ldots \ldots \ldots \ldots \ldots \ldots
\end{array}
$$

where $L_{d}$ and $e_{q}$ represent the inductance on d-axis and the electromotive force of the motor. The Class-D power amplifiers output the voltages $v_{u}, v_{v}, v_{w}$ to drive the linear motor according to the 3-phase voltage references as follows:

$$
\left[\begin{array}{c}
v_{u} \\
v_{v} \\
v_{w}
\end{array}\right]=\left[\begin{array}{ccc}
L^{\prime} s+R^{\prime} & -M^{\prime} s / 2 & -M^{\prime} s / 2 \\
-M^{\prime} s / 2 & L^{\prime} s+R^{\prime} & -M^{\prime} s / 2 \\
-M^{\prime} s / 2 & -M^{\prime} s / 2 & L^{\prime} s+R^{\prime}
\end{array}\right]\left[\begin{array}{c}
i_{u} \\
i_{v} \\
i_{w}
\end{array}\right]+\left[\begin{array}{c}
e_{u} \\
e_{v} \\
e_{w}
\end{array}\right]
$$

where $L^{\prime}, R^{\prime}$, and $M^{\prime}$ denote the inductance, resistance, and the mutual inductance of the motor. $e_{u}, e_{v}, e_{w}$ are the back electromotive force.

4.2 Current Noise of a Class-D Power Amplifier In a Class-D power amplifier, the current noise occurs due to PWM voltage generated by the inverter. The amplitude of the current noise, which affects the outer force control system, is calculated in this section.

The PWM voltage shown in Fig. 5(a) is expressed by Fourier series as follows:

$$
v_{q}(t)=V_{d c} \frac{T_{o n}}{T_{c}}
$$




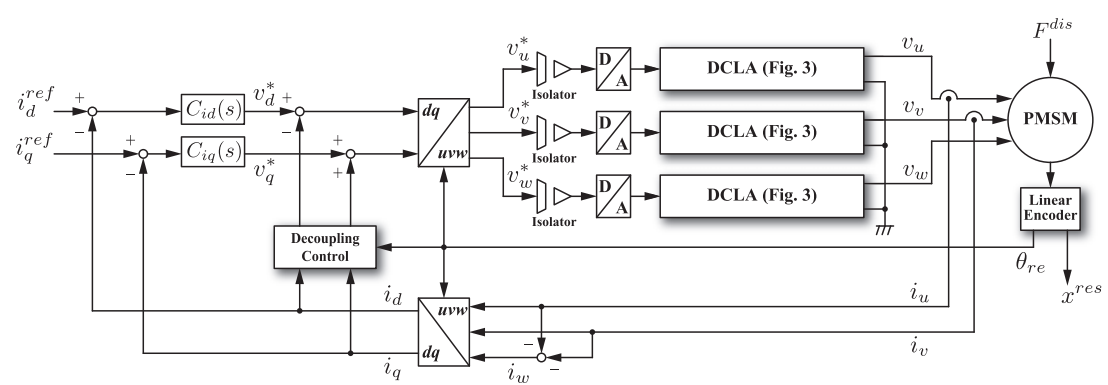

Fig. 6. Block diagram of current control system with DCLAs

$$
\begin{aligned}
& +\frac{2 V_{d c}}{\omega_{0} T_{c}} \sum_{n \in N} \frac{1}{n}\left[\sin \left(n w_{0}\left(T_{o n}-t\right)\right)+\sin n w_{0} t\right] \\
& \ldots \ldots \ldots \ldots \ldots \ldots(11) \\
& \omega_{0}=2 \pi f_{c} \\
& T_{\text {on }}=\frac{v_{q}^{*} T_{c}}{V_{d c}} .
\end{aligned}
$$

where $T_{o n}$ and $T_{c}$ denote the pulse width and the period of PWM carrier, respectively. $f_{c}$ denotes the PWM carrier frequency, thereby, $T_{c}=1 / f_{c}$. $n$ denotes the order of Fourier series.

From (8) and (10), the equivalent circuit on the d-q coordinate is shown in Fig. 5(b). The impedance $Z\left(j n \omega_{0}\right)$ of the $\mathrm{R}-\mathrm{L}$ circuit shown in Fig. 5(b) is given by

$$
Z\left(j n \omega_{0}\right)=R_{q}+j n \omega_{0} L_{q} .
$$

Then, q-axis current $i_{q}(t)$ is derived as

$$
\begin{aligned}
\left.i_{q}(t)\right|_{\text {const } v_{q}^{*}}=\frac{v_{q}(t)}{Z\left(j n \omega_{0}\right)} \\
=\frac{V_{d c}}{R_{q}} \frac{T_{o n}}{T_{c}}+\frac{2 V_{d c}}{\omega_{0} T_{c}} \sum_{n \in N} \frac{1}{n \sqrt{R_{q}^{2}+n^{2} \omega_{0}^{2} L_{q}^{2}}} \\
\quad\left[\sin \left(n \omega_{0}\left(T_{o n}-t\right)+\tan ^{-1} \frac{n \omega_{0} L_{q}}{R_{q}}\right)\right. \\
\left.+\sin \left(n \omega_{0} t-\tan ^{-1} \frac{n \omega_{0} L_{q}}{R_{q}}\right)\right] \ldots \ldots
\end{aligned}
$$

The second term of the q-axis current derived as (15) represents the noise current. Furthermore, the maximum and minimum value of the current noise on q-axis $i_{\text {qmax }}, i_{\text {qmin }}$ are calculated as

$$
\begin{aligned}
& i_{\text {qmax }}=i_{q}\left(T_{o n}\right) \\
& i_{\text {qmin }}=i_{q}(0) .
\end{aligned}
$$

Since the linear motor, which a force constant is defined as $K_{f}$, generates the force $F(t)$ depending on $F(t)=K_{f} i_{q}(t)$, the force noise occurs due to the current noise. Hence, the fine force control cannot be realized in this case.

Besides, the current on d-axis $i_{d}(t)$ is used for the decoupling control calculated as (8). Therefore, the modified voltage reference on q-axis $v_{q}^{* \prime}$ is given by

$$
v_{q}^{* \prime}=v_{q}^{*}+L_{d} \dot{\theta}_{r e}\left[\frac{v_{q}^{*}}{R_{d}}+\frac{2 V_{d c}}{\omega_{0} T_{c}} \sum_{n \in N} \frac{1}{n \sqrt{R_{d}^{2}+n^{2} \omega_{0}^{2} L_{d}^{2}}}\right.
$$

$$
\begin{aligned}
& \left\{\sin \left(n w_{0}\left(T_{o n}-t\right)+\tan ^{-1} \frac{n \omega_{0} L_{d}}{R_{d}}\right)\right. \\
& \left.\left.\quad+\sin \left(n \omega_{0} t-\tan ^{-1} \frac{n \omega_{0} L_{d}}{R_{d}}\right)\right\}\right]+e_{q} \cdots \cdots
\end{aligned}
$$

where $R_{d}$ denotes the resistance on d-axis. The d-axis current noise caused by the PWM or PDM operations is injected to the voltage reference on q-axis. As with the current on q-axis, the $\mathrm{d}$-axis noise should be also reduced.

\subsection{Low-Noise Current Control using DCLAs}

Figure 6 depicts the current control system using DCLAs. Fundamentally, the calculations of the current control system shown in Fig. 6 is same as the control method mentioned in Sect. 4.1. However, three DCLAs are used instead of the Class-D amplifiers in order to realize the low-noise current control. Compared with the Class-D amplifiers, DCLAs are capable of generating very smooth 3-phase voltage according to the voltage references $v_{u}^{*}, v_{v}^{*}, v_{w}^{*}$. In addition, the q-axis current provided by DCLAs is derived as

$$
\left.i_{q}(t)\right|_{\text {const } v_{q}^{*}}=\frac{v_{q}(t)}{Z(j n \omega 0)}=\frac{v_{q}^{*}}{R_{q}} .
$$

Furthermore, the maximum and minimum value of the q-axis noise current are reduced as follows:

$$
i_{\text {qmax }}=i_{\text {qmin }}=\frac{v_{q}^{*}}{R_{q}}
$$

In (19), the q-axis current $i_{q}(t)$ is constant on condition that the voltage reference $v_{q}^{*}$ is also constant. Hence, the current noise derived as (15)-(17) disappear by using DCLAs.

The voltage/current control systems including DCLAs has been realized by analog circuits, which were presented in Ref. (32). As mentioned in Sect. 3.1, this paper implements the voltage control system using analog circuits. On the other hand, the current control system is realized by a FPGA.

4.4 Sensorless Force Control using 1st-order Disturbance Observer The sensorless force control with the local current control system mentioned above is explained.

Figure 7 shows a block diagram of the force control system based on a 1 st-order disturbance observer ${ }^{(33)-(35)} . M_{n}$ and $K_{f n}$ denote the nominal mass and the nominal force constant of the linear motor, respectively. $Z_{e}(s)$ represents environmental mechanical impedance (target object). When the end effector of the linear motor is contacting with the surface of the object, the target object generates disturbance force $F^{d i s}$. As shown in Fig. 7, the 1st-order disturbance observer estimates the disturbance force $\hat{F}^{d i s}$, and cancels the disturbance, so 


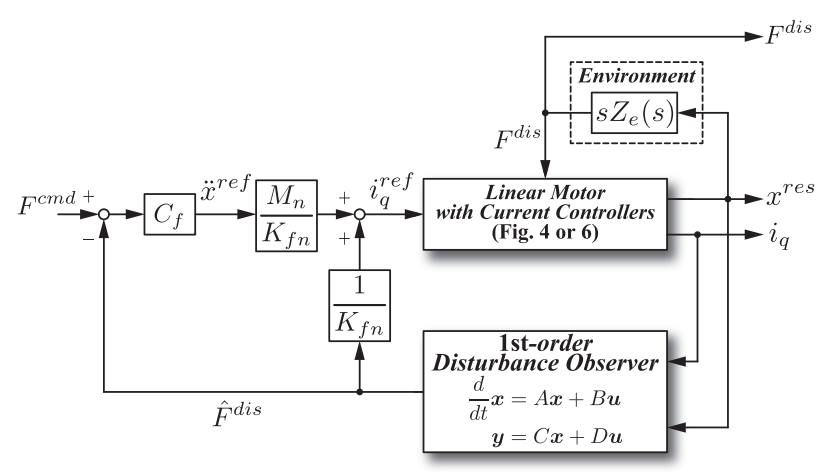

Fig. 7. Block diagram of force control system based on disturbance observer

that the acceleration control is realized ${ }^{(33)-(35)}$.

The state equations of the 1 st-order disturbance observer is as follows:

$$
\begin{aligned}
\frac{d}{d t} \boldsymbol{x} & =A \boldsymbol{x}+B \boldsymbol{u} \\
\boldsymbol{y} & =C \boldsymbol{x}+D \boldsymbol{u} \\
\boldsymbol{u} & =\left[\begin{array}{ll}
i_{q}(t) & x^{r e s}(t)
\end{array}\right]^{T} \\
A & =\left[\begin{array}{ll}
0 & -k_{1} \\
1 & -k_{2}
\end{array}\right], B=\left[\begin{array}{cc}
0 & -k_{1} k_{2} \\
\frac{K_{f n}}{M_{n}} & \left(k_{1}-k_{2}^{2}\right)
\end{array}\right] \\
C & =\left[\begin{array}{ll}
-M_{n} & 0
\end{array}\right], D=\left[\begin{array}{cc}
0 & -k_{1} M_{n}
\end{array}\right] \\
\boldsymbol{y} & =\hat{F}^{d i s}(t) \ldots \ldots \ldots \ldots \ldots \ldots \ldots \ldots \ldots \ldots \ldots \ldots \ldots
\end{aligned}
$$

where $k_{1}$ and $k_{2}$ represent free parameters. Then, the transfer function matrix of the 1st-order disturbance observer $G_{d o}(s)$ is given by

$$
\begin{aligned}
G_{d o}(s) & =C(s \boldsymbol{I}-A)^{-1} B+D \\
& =\frac{k_{1}}{s^{2}+k_{2} s+k_{1}}\left[\begin{array}{ll}
K_{f n} & -M_{n} s^{2}
\end{array}\right] .
\end{aligned}
$$

(22) is rewritten as

$$
\hat{F}^{d i s}(s)=\frac{g_{d i s}^{2}}{s^{2}+\frac{g_{d i s}}{Q_{d i s}} s+g_{d i s}{ }^{2}}\left[\begin{array}{lll}
K_{f n} & -M_{n} s^{2}
\end{array}\right] \mathcal{L}^{-1}\{\boldsymbol{u}\}
$$

where $g_{\text {dis }}$ and $Q_{\text {dis }}$ denote bandwidth and quality factor of the 1 st-order disturbance observer. When $Q_{d i s}=0.5$, the free parameters $k_{1}, k_{2}$ are determined by

$$
\begin{aligned}
& k_{1}=g_{\text {dis }}{ }^{2} \\
& k_{2}=2 g_{\text {dis }} .
\end{aligned}
$$

According to (23), the 1st-order disturbance observer is equivalent to a 2 nd-order LPF relevant to the force. Thus, the disturbance observer is capable of detecting the force response without any force sensors. Furthermore, a pseudo differentiator required for a 0 -order disturbance observer is not required. Therefore, compared with the 0 -order disturbance observer, the level of detected noise can be reduced.

In order to control the force of the linear motor, the acceleration reference $\ddot{x}^{r e f}$ is calculated as

$$
\ddot{x}^{r e f}=C_{f}\left(F^{c m d}-\hat{F}^{d i s}\right)
$$

where $F^{c m d}$ denotes the force command. $C_{f}$ represents a
Table 4. Setup parameters of simulations and experiment

\begin{tabular}{lc|rl}
\hline \hline Parameters & Symbols & Value & Units \\
\hline Resistance of linear motor on d-q axis & $R_{d}=R_{q}$ & 4.5 & $\Omega$ \\
Inductance of linear motor on d-q axis & $L_{d}=L_{q}$ & 0.98 & $\mathrm{mH}$ \\
Force constant & $K_{f}$ & 3.0 & $\mathrm{~N} / \mathrm{A}$ \\
Mass of actuator & $M$ & 0.15 & $\mathrm{~kg}$ \\
Encoder resolution & $x_{\text {reso }}$ & 100 & $\mathrm{~nm}$ \\
Sampling frequency & $f_{s}$ & 30 & $\mathrm{kHz}$ \\
Sampling time & $T_{s}$ & 33.3 & $\mu \mathrm{s}$ \\
Carrier frequency of PWM (Class-D) & $f_{c}$ & 15 & $\mathrm{kHz}$ \\
Bandwidth of PI current controllers & $\omega_{c}$ & 6280 & $\mathrm{rad} / \mathrm{s}$ \\
Bandwidth of disturbance observer & $g_{d i s}$ & 1000 & $\mathrm{rad} / \mathrm{s}$ \\
Gain of force controller & $C_{f}$ & 3.0 & $\mathrm{~kg}$ \\
Conversion time of A/D converters (AD7899) & & 2.5 & $\mu \mathrm{s}$ \\
Settling time of D/A converters (AD9764) & & 35 & $\mathrm{~ns}$ \\
\hline
\end{tabular}

force controller, which is implemented as simple P-control in this paper. The current control system shown in Figs. 4 and 6 drives the linear motor according to the d-q axes current references, which are calculated as

$$
\begin{aligned}
& i_{d}^{r e f}=0 \ldots \ldots \ldots \ldots \ldots \\
& i_{q}^{r e f}=\frac{M_{n}}{K_{f n}} \ddot{x}^{r e f}+\frac{1}{K_{f n}} \hat{F}^{d i s}
\end{aligned}
$$

where $K_{f n}$ and $M_{n}$ represent the nominal force constant and the nominal mass of the linear actuator, respectively.

In the force control system mentioned above, the transfer function from the force command to the force response $G_{f}(s)$ is given by

$$
\begin{aligned}
G_{f}(s) & =\frac{F^{d i s}(s)}{F^{c m d}(s)} \\
& =\frac{C_{f} Z_{e}(s)}{s+\frac{Z_{e}(s)}{M_{n}}\left(G_{d o}(s)\left(C_{f} M_{n}-1\right)+1\right)} .
\end{aligned}
$$

By final-value theorem,

$$
\lim _{s \rightarrow 0} G_{f}(s)=1 \text {. }
$$

Hence, the robust force control is achieved.

In addition, the force generated by the force control system is given by

$$
F^{d i s}(t)=K_{f} \mathcal{L}^{-1}\left\{\frac{Z_{e}(s)}{M s+Z_{e}(s)} \mathcal{L}\left\{i_{q}(t)\right\}\right\} \cdots
$$

where $K_{f}$ and $M$ denote the force constant and the inertia of the linear motor. The force noise is derived from (15) and (31) when the Class-D amplifiers are used to drive the motor as follows:

$$
\begin{aligned}
& \left.F^{d i s}(t)\right|_{\text {const } v_{q}^{*}}=K_{f} \mathcal{L}^{-1}\left\{\frac { Z _ { e } ( s ) } { M s + Z _ { e } ( s ) } \left(\frac{V_{d c}}{R_{q}} \frac{T_{o n}}{T_{c}}\right.\right. \\
& +\frac{2 V_{d c}}{\omega_{0} T_{c}} \sum_{n \in N} \frac{1}{n \sqrt{R_{q}^{2}+n^{2} \omega_{0}^{2} L_{q}^{2}}} \frac{1}{s^{2}+n^{2} \omega_{0}^{2}} \\
& {\left[s\left\{\sin \left(n \omega_{0} T_{o n}-\varphi_{n}\right)+\sin \varphi_{n}\right\}\right.} \\
& \left.\left.\left.-n \omega_{0}\left\{\cos \left(n \omega_{0} T_{o n}-\varphi_{n}\right)+\cos \varphi_{n}\right\}\right]\right)\right\} \text {, } \\
& \varphi_{n}=-\tan ^{-1} \frac{n \omega_{0} L_{q}}{R_{q}} .
\end{aligned}
$$




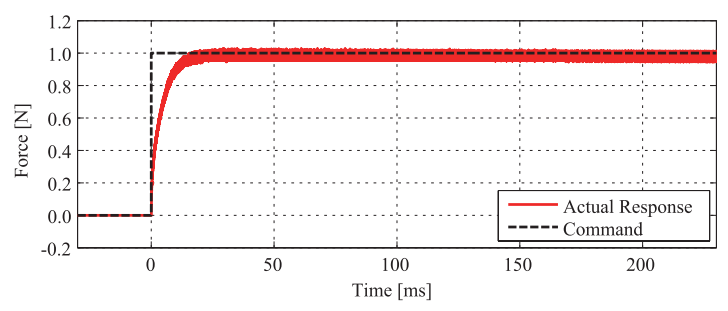

(a) Force command and actual response

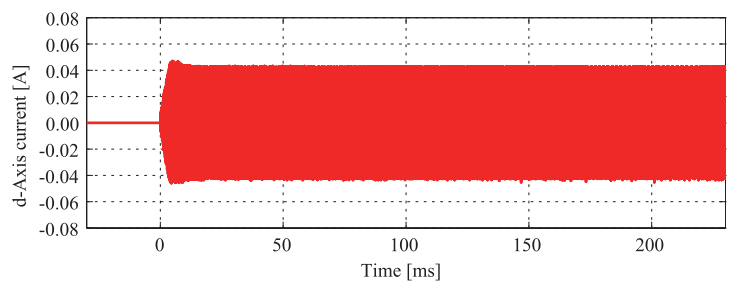

(b) d-axis current response

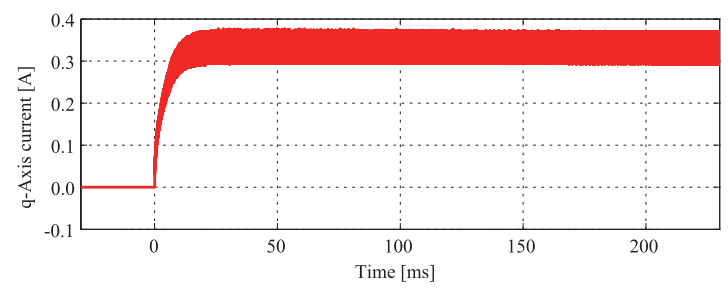

(c) q-axis current response

Fig. 8. Simulation results. (Class-D power amplifiers.)

Thus, the current noise on q-axis should be decreased to reduce the force noise represented by (32). In particular, the force noise increases in the case of a high-power direct drive actuator, which the force constant $K_{f}$ is very high.

On the other hand, the force generated by DCLAs is derived as

$$
\left.F^{d i s}(t)\right|_{\text {const } v_{q}^{*}}=K_{f} \frac{v_{q}}{R_{q}} \mathcal{L}^{-1}\left\{\frac{Z_{e}(s)}{M s+Z_{e}(s)}\right\}
$$

For that reason, DCLAs are suitable for the low-noise and fine force control.

4.5 Permissible Current Disturbance This paper also studies about "permissible current disturbance."

In Fig. 7, the transfer function $G_{i q x}(s)$ from $i_{q}$ to $x^{\text {res }}$ is given by

$$
G_{i q x}(s)=\frac{x^{r e s}}{i_{q}}=\frac{K_{f}}{M s^{2}+Z_{e}(s) s} .
$$

This study assumes that the target environment is composed of the stiffness $K_{e}$ as follows:

$$
Z_{e}(s)=\frac{K_{e}}{s}
$$

Assuming the force command is constant value, $i_{q}$ is also constant in a steady-state. Thereby, displacement of a motor $x^{\text {res }}$ is given by

$$
x^{r e s}=\lim _{s \rightarrow 0} G_{i q x}(s) i_{q}=\frac{K_{f}}{K_{e}} i_{q} .
$$

Therefore, an encoder(position sensor) is capable of detecting the displacement affected by $i_{q}$. The minimal current, which can be detected by the encoder, is calculated as

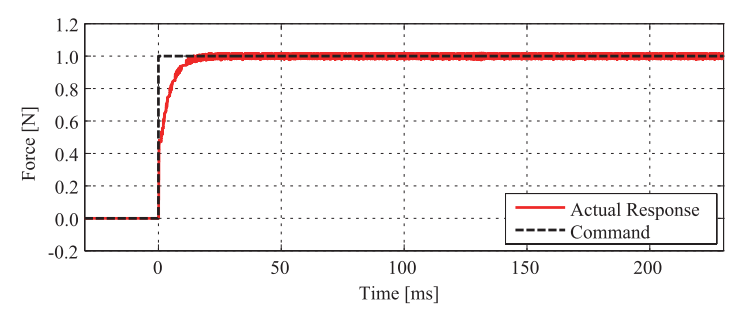

(a) Force command and actual response

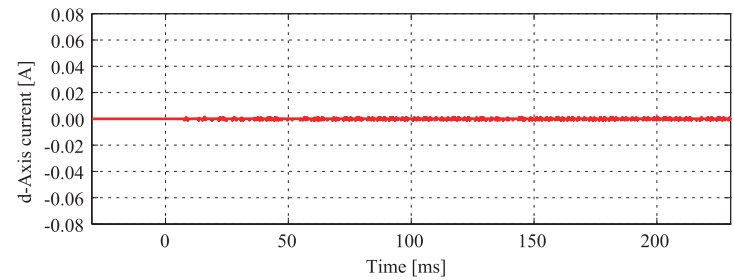

(b) d-axis current response

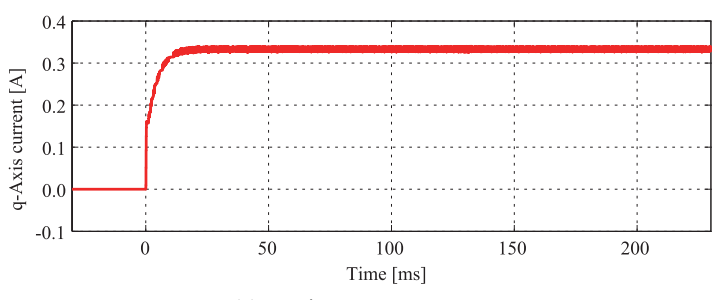

(c) q-axis current response

Fig. 9. Simulation results. (DCLAs)

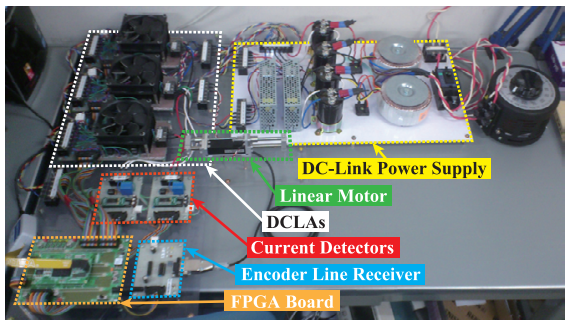

Fig. 10. Experimental setup

$$
i_{q P C D}=x^{r e s o} \frac{K_{e}}{K_{f}}
$$

where $x^{\text {reso }}$ denotes the resolution of the encoder. In case that the disturbance current is larger than $i_{q P C D}$, the encoder becomes noise source affecting the disturbance observer. Therefore, the following equation should be satisfied.

$$
\left.\left|i_{q}^{r e f}-i_{q}\right|<i_{q P C D} \quad \text { ( const } i_{q}^{r e f}\right)
$$

In this paper, $i_{q P C D}$ which is represented by (38) is defined as minimum "permissible current disturbance." The permissible current disturbance is one of the candidates for indicating the required quality of a amplifier for the force control.

\section{Simulations}

5.1 Simulation Conditions The numerical simulations of the force control system shown in Fig. 7 are conducted. In the simulations of the force control using Class-D power amplifiers, the voltage for driving the linear motor is generated by PWM. On the other hand, the transfer function of DCLAs from the input voltage $v_{i}$ to the output voltage $v_{o}$ is modeled as 


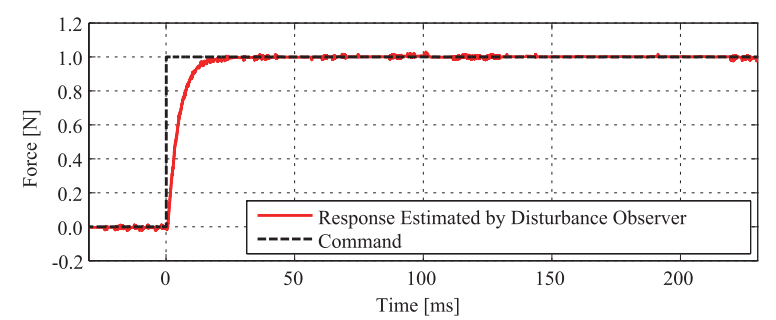

(a) Force command and actual response

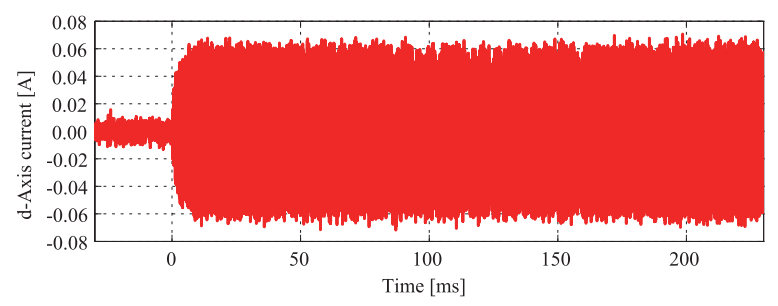

(b) d-axis current response

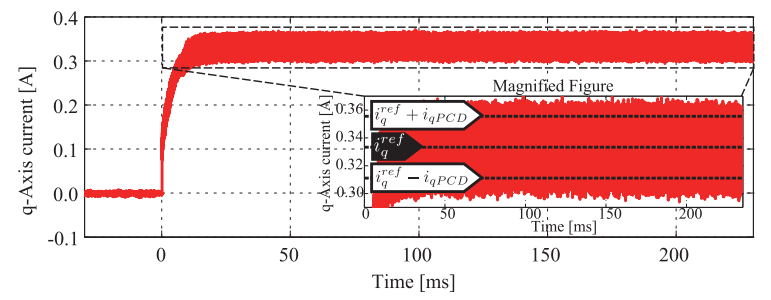

(c) q-axis current response

Fig. 11. Experimental results. (Class-D power amplifiers.)

$$
\begin{aligned}
& {\left[\begin{array}{c}
v_{u} \\
v_{v} \\
v_{w}
\end{array}\right]=G_{a m p}(s) \boldsymbol{I}_{3}\left[\begin{array}{c}
v_{u}^{*} \\
v_{v}^{*} \\
v_{w}^{*}
\end{array}\right] \ldots \ldots \ldots \ldots \ldots \ldots \ldots \ldots \ldots \ldots \ldots \ldots \ldots}
\end{aligned}
$$

where $g_{a m p}$ and $T_{s}$ denote the bandwidth of DCLAs and the control period. $g_{a m p}$ is set to $30 \mathrm{kHz}$ in the simulations. Table 4 describes the parameters of the plant systems and the controllers.

5.2 Simulation Results Figures 8 and 9 represent the simulation results of the force control system using the Class$\mathrm{D}$ and DCLAs. In the Class-D power amplifiers, the force noise and the current noise occur due to the PWM. By using DCLAs, the force noise and the current noise are reduced.

\section{Experiment}

To verify the validity of the force control using DCLAs, the comparative experiments are carried out in this paper.

6.1 Experimental Setup Figure 10 shows the experimental setup, which consists of the 3-phase DCLAs, the linear motor, and the power supplies. In the equipment, n-channel and p-channel MOSFETs are 2SK1530 and 2SJ201, which are complementary type. The FPGA for processing the force control and operating DCLAs is ALTERA EP3C55F780C8N which has 55,856 Logic Elements (LE) and 156 multipliers. The material of the target object is an aluminum block. In this experiment, initial position of the end effector of the linear motor is set to surface of the target object. As with the simulations, Table 4 shows the parameters of the linear motor, the current controllers, and the force control system.

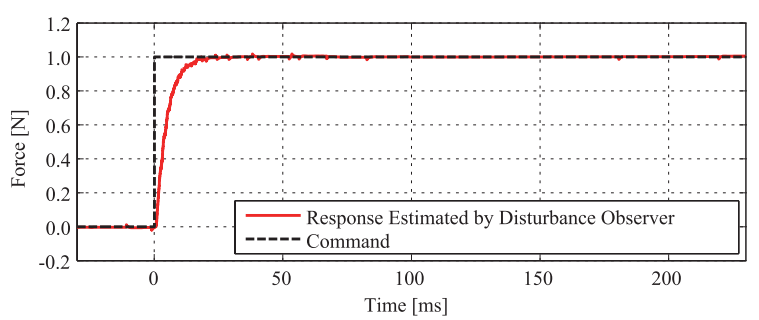

(a) Force command and actual response

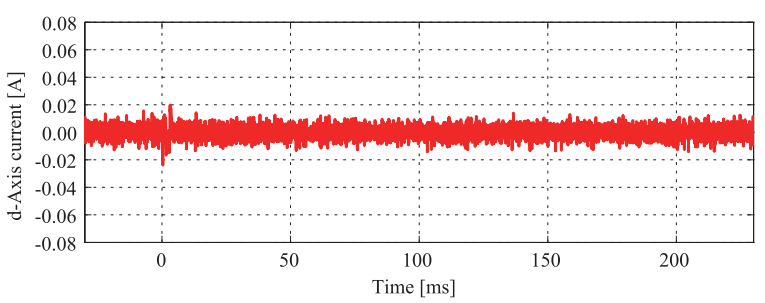

(b) d-axis current response

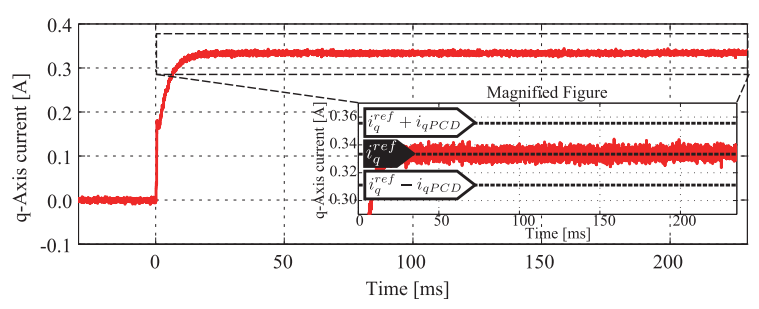

(c) q-axis current response

Fig. 12. Experimental results. (DCLAs)

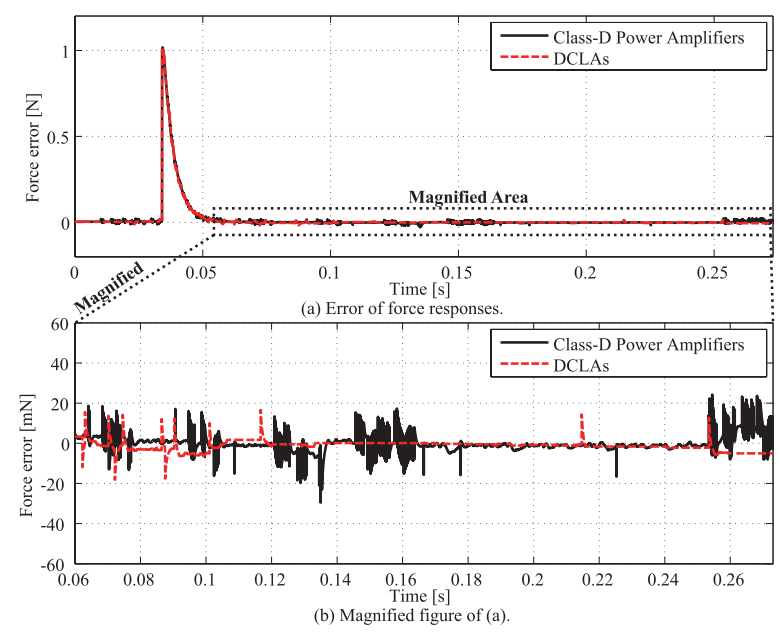

Fig. 13. Error evaluations of force responses

\subsection{Experimental Results}

6.2.1 Time Responses Figure 11 represents the experimental results of the force control using the general PWM inverter, which is categorized as Class-D power amplifiers. As shown in the results, the current noise on $\mathrm{d}-\mathrm{q}$ coordinates is observed. The disturbance observer is equivalent to a lowpass-filter with respect to the force response. Therefore, the force noise cannot be blocked completely when the very large noise occurs. The noise generated by the power amplifiers is attenuated and passed actually, although the cut-off frequency is set as $1000 \mathrm{rad} / \mathrm{s}$. As a results, the residual force noise affects the accuracy of the force generation.

Figure 12 shows the experimental results of the force control using DCLAs developed in this research. Compared with the Class-D power amplifiers, the current noise on $\mathrm{d}-\mathrm{q}$ 


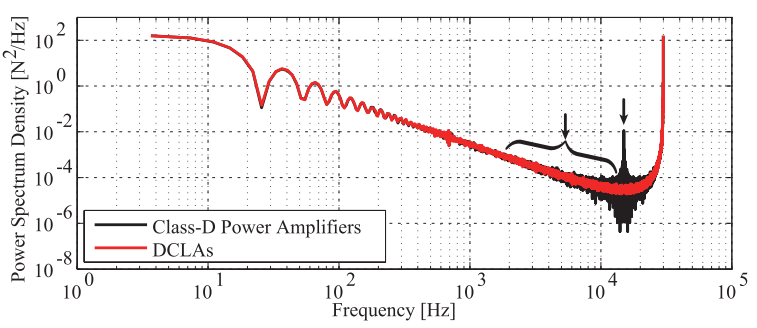

(a) Force responses shown in Figs. 11(a) and 12(a)

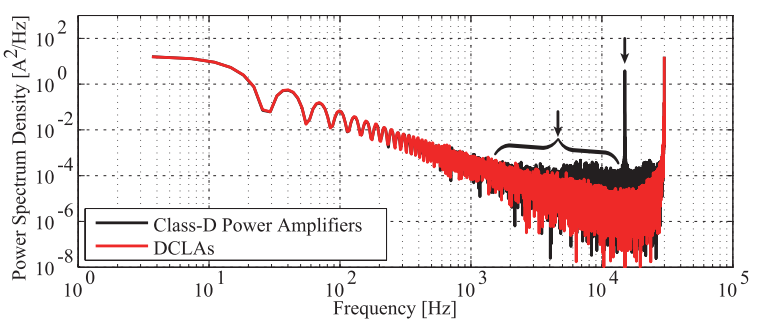

(b) q-axis current responses shown in Figs. 11(c) and 12(c)

Fig. 14. Power spectral densities

coordinates is reduced.

Figure 13 shows the error of force responses. As shown in the results, the force error generated by the force control system with DCLAs is reduced compared with the Class-D amplifier.

6.2.2 Permissible Current Disturbance In this experiment, the permissible current disturbance defined by (38) is calculated as

$$
\begin{aligned}
i_{q P C D} & =x^{\text {reso }} \frac{K_{e}}{K_{f}}=100[\mathrm{~nm}] \times \frac{6.67 \times 10^{5}[\mathrm{~N} / \mathrm{m}]}{3.0[\mathrm{~N} / \mathrm{A}]} \\
& \simeq 22[\mathrm{~mA}] . \cdots \ldots \ldots \ldots \ldots \ldots \ldots \ldots \ldots \ldots \ldots \ldots \ldots
\end{aligned}
$$

The stiffness $K_{e}$ of the aluminum block is measured by the pre-experiment. Figures 11(c) and 12(c) show the current responses on q-axis and the permissible current disturbance. When DCLAs drive the motor, (39) is satisfied differently from the Class-D amplifiers.

6.2.3 Power Spectral Densities In order to evaluate the force responses of the Class-D and DCLAs, the power spectral density of the force estimated by the disturbance observer is calculated. The power spectral density $P_{d}$ is given by

$$
P_{d}[k]=\frac{\phi[k] \phi^{*}[k]}{N_{m}}
$$

where $N_{m}$ and $k$ denote number of the force data and frequency, respectively. The superscript $*$ indicates complex conjugate. $\phi$ is calculated as

$$
\phi[k]=\sum_{m=1}^{N_{m}} \hat{F}^{d i s}[m] \exp \left\{\frac{-j 2 \pi(k-1)(m-1)}{N_{m}}\right\} \ldots
$$

where $m$ denotes discrete time. In this paper, the power spectral density of the q-axis current is also calculated.

Figure 14(a) represents the power spectral density of the force responses and of the q-axis current responses. As shown in Fig. 14(b), the force noise is drastically reduced, compared to the general PWM inverter.

6.2.4 Power Efficiency In order to calculate the
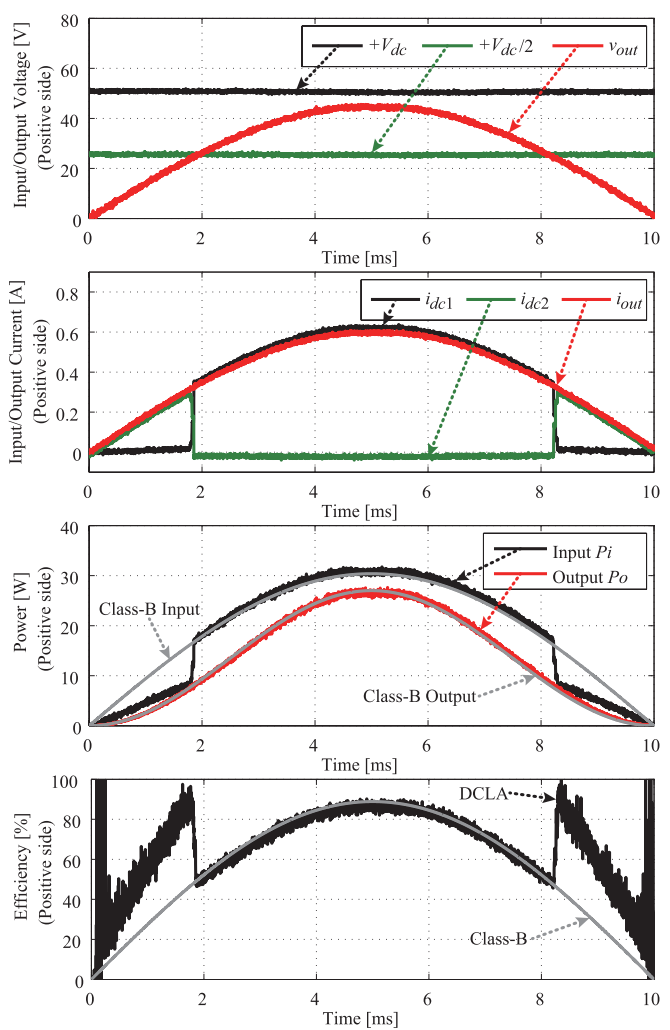

Fig. 15. Experimental results of DCLA. (Point of Measurements is shown in Fig. 3.)

power losses and efficiency, the input DC voltages, output voltage, input/output current, input/output power, and efficiency of DCLA and the Class-D amplifier are measured by the experiment. Figure 15 shows the experimental results of DCLA. In this experiment, the input DC voltage $V_{d c}$ is set as $50 \mathrm{~V}$, the output voltage $v_{\text {out }}$ is set to $45 \sin (2 \pi f t)$ [V], and the frequency $f$ is set as $50 \mathrm{~Hz}$. In Fig. 15, the gray lines show the theoretical power and efficiency of a Class-B amplifier. The power loss of DCLA is lower than the Class-B linear amplifier. Moreover, in this situation, the power efficiency of Class-B, DCLA, and Class-D amplifiers is calculated as $\eta_{b}=69.8 \%, \eta_{D C L A}=74.2 \%$, and $\eta_{d}=89.0 \%$, respectively. (Due to the limitation of the output voltage of the operational amplifier stage in front of gate pins, the efficiency is lower than the theoretical efficiency. Therefore, a "rail-to-rail" high voltage operational amplifier or another method have to be developed newly to overcome this problem in future.) In short, this comparisons confirm that $\eta_{b}<\eta_{D C L A}<\eta_{d}$.

\section{Conclusions}

In this paper, DCLAs for a low-noise and fine force control were developed. The typical Class-B and Class-D power amplifiers are not suitable for driving a motor, due to the power efficiency and switching noise. In particular, a force control system using the Class-D power amplifiers cannot generate low-noise and fine force because of PWM. On the other hand, by using DCLAs, the problems such as the power efficiency, the current noise, and the force noise were solved.

By the theoretical analysis, the simulations, and the experiment, the validity of the force control using DCLAs was verified. The low-noise and fine-efficiency motor drivers will 
be applied to precise control, numerical controlled machine tools, industrial and medical robots, and real-world haptics. In particular, the presented approach will be useful for the very precise force control in the industrial and medical field.

\section{References}

( 1 ) M. Mezaroba, D.C. Martins, and I. Barbi: "A ZVS PWM Half-Bridge Voltage Source Inverter With Active Clamping", IEEE Trans. Industrial Electronics, Vol.54, No.5, pp.2665-2672 (2007)

( 2 ) A. Boglietti, R. Bojoi, A. Cavagnino, and A. Tenconi: "Efficiency Analysis of PWM Inverter Fed Three-Phase and Dual Three-Phase High Frequency Induction Machines for Low/Medium Power Applications", IEEE Trans. Industrial Electronics, Vol.55, No.5, pp.2015-2023 (2008)

( 3 ) D.S. Zinger and T. Lee: "Performance of MCT's in a current-regulated AC/DC PDM converter", IEEE Trans. Power Electronics, Vol.11, No.1, pp.49-56 (1996)

(4) K. Szabat and T. Orlowska-Kowalska: "Application of the Kalman Filters to the High-Performance Drive System With Elastic Coupling", IEEE Trans. Industrial Electronics, Vol.59, No.11, pp.4226-4235 (2012)

( 5 ) L. Idkhajine, E. Monmasson, and A. Maalouf: "Fully FPGA-Based Sensorless Control for Synchronous AC Drive Using an Extended Kalman Filter", IEEE Trans. Industrial Electronics, Vol.59, No.10, pp.3908-3918 (2012)

( 6 ) B. Veselic, B. Perunicic-Drazenovic, and C. Milosavljevic: "HighPerformance Position Control of Induction Motor Using Discrete-Time Sliding-Mode Control", IEEE Trans. Industrial Electronics, Vol.55, No.11, pp.3809-3817 (2008)

( 7 ) B. Veselic, B. Perunicic-Drazenovic, and C. Milosavljevic: "Improved Discrete-Time Sliding-Mode Position Control Using Euler Velocity Estimation”, IEEE Trans. Industrial Electronics, Vol.57, No.11, pp.3840-3847 (2010)

( 8 ) C. Mademlis and I. Kioskeridis: "Gain-Scheduling Regulator for HighPerformance Position Control of Switched Reluctance Motor Drives", IEEE Trans. Industrial Electronics, Vol.57, No.9, pp.2922-2931 (2010)

( 9 ) J. Wu, Z. Xiong, K-M. Lee, and H. Ding: "High-Acceleration Precision Point-to-Point Motion Control With Look-Ahead Properties", IEEE Trans. Industrial Electronics, Vol.58, No.9, pp.4343-4352 (2011)

(10) T. Stepien, L. Sweet, M. Good, and M. Tomizuka: "Control of tool/workpiece contact force with application to robotic deburring", IEEE Journal of Robotics and Automation, Vol.3, No.1, pp.7-18 (1987)

(11) T. Insperger, L.L. Kovacs, P. Galambos, and G. Stepan: "Increasing the Accuracy of Digital Force Control Process Using the Act-and-Wait Concept", IEEE/ASME Trans. Mechatronics, Vol.15, No.2, pp.291-298 (2009)

(12) J. Park, D. Hyun, W.H. Cho, T.H. Kim, and H.S. Yang: "Normal-Force Control for an In-Pipe Robot According to the Inclination of Pipelines", IEEE Trans. Industrial Electronics, Vol.58, No.12, pp.5304-5310 (2011)

(13) C. Mitsantisuk, K. Ohishi, and S. Katsura: "Control of Interaction Force of Twin Direct-Drive Motor System Using Variable Wire Rope Tension With Multisensor Integration", IEEE Trans. Industrial Electronics, Vol.59, No.1, pp.498-510 (2012)

(14) H-W. Chow and N.C. Cheung: "Disturbance and Reponse Time Improvement of Submicrometer Precision Linear Motion System by Using Modified Disturbance Compensator and Internal Model Reference Control", IEEE Trans. Industrial Electronics, Vol.60, No.1, pp.139-150 (2013)

(15) T.T. Phuong, R. Furusawa, M. Nandayapa, C. Mitsantisuk, and K. Ohishi: "FPGA-based Wideband Force Control System with Friction-Free and NoiseFree Force Observation", IEEJ Journal of Industry Applications, Vol.1, No.3, pp.178-190 (2012)

(16) R.J. Anderson and M.W. Spong: "Bilateral Control of Teleoperators with Time Delay", IEEE Trans. Automatic Control, Vol.34, No.5, pp.494-501 (1989)

(17) D.A. Lawrence: "Stability and transparency in bilateral teleoperation", IEEE Trans. Robotics and Automation, Vol.9, No.5, pp.624-637 (1993)

(18) C. Mitsantisuk, K. Ohishi, and S. Katsura: "Estimation of Action/Reaction Forces for the Bilateral Control Using Kalman Filter", IEEE Trans. Industrial Electronics, Vol.59, No.11, pp.4383-4393 (2012)

(19) H. Tanaka and K. Ohnishi: "Haptic data compression/decompression using DCT for motion copy system", IEEE International Conference on Mechatronics, ICM 2009, pp.1-6 (2009)

(20) Y. Yokokura, S. Katsura, and K. Ohishi: "Stability Analysis and Experimental Validation of a Motion-Copying System", IEEE Trans. Industrial Electronics, Vol.56, No.10, pp.3906-3913 (2009)
(21) H. Kuwahara, T. Shimono, H. Tanaka, D. Yashiro, and K. Ohnishi: "Abstraction of Action Components Unconstrained by Alignment of Haptic Sensing Points", IEEE Trans. Industrial Electronics, Vol.58, No.8, pp.3196-3204 (2011)

(22) N. Tsunashima and S. Katsura: "Spatiotemporal Coupler: Storage and Reproduction of Human Finger Motions", IEEE Trans. Industrial Electronics, Vol.59, No.2, pp.1074-1085 (2012)

(23) T. Nozaki, T. Mizoguchi, and K. Ohnishi: "Real-World Haptics for Motion Realization”, IEEJ Journal of Industry Applications, Vol.2, No.1, pp.1-6 (2013)

(24) T. Sampei, S. Ohashi, Y. Ohta, and S. Inoue: "Highest Efficiency and Super Quality Audio Amplifier using MOS Power FETS in Class G Operation", IEEE Trans. Consumer Electronics, Vol.24, No.3, pp.300-307 (1978)

(25) F.H. Raab: "Average Efficiency of Class-G Power Amplifiers", IEEE Trans. Consumer Electronics, Vol.32, No.2, pp.145-150 (1986)

(26) A.D. Downey and G.M. Wierzba: "A Class-G/FB Audio Amplifier", IEEE Trans. Consumer Electronics, Vol.53, No.4, pp.1537-1545 (2007)

(27) A.D. Downey and G.M. Wierzba: "A Class-G Headphone Amplifier in $65 \mathrm{~nm}$ CMOS Technology", IEEE Journal of Solid-State Circuits, Vol.45, No.12, pp.2530-2542 (2010)

(28) S. Carabelli, F. Maddaleno, and M. Muzzarelli: "High-efficiency linear power amplifier for active magnetic bearings", IEEE Trans. Industrial Electronics, Vol.47, No.1, pp.17-24 (2000)

(29) H. Fujita: "A High-Efficiency Diode-Clamped Linear Amplifier", IEEJ Trans. Industry Applications, Vol.127, No.1, pp.9-17 (2007)

(30) H. Fujita and N. Yamashita: "Performance of a Diode-Clamped Linear Amplifier", IEEE Trans. Power Electronics, Vol.23, No.1, pp.824-831 (2008)

(31) H. Fujita: "Emerging Technologies for Multilevel Converters in Japan", IEEJ Journal of Industry Applications, Vol.1, No.2, pp.95-101 (2012)

(32) H. Fujita: "A Single-Phase Utility-Interface Circuit Without Any AC Inductor nor EMI Filter", IEEE Trans. Industry Applications, Vol.45, No.5, pp.1860-1867 (2009)

(33) K. Ohnishi, M. Shibata, and T. Murakami: "Motion Control for Advanced Mechatronics", IEEE/ASME Trans. Mechatronics, Vol.1, No.1, pp.56-67 (1996-3)

(34) T. Murakami, F. Yu, and K. Ohnishi: "Torque Sensorless Control in Multidegree-of-freedom Manipulator”, IEEE Trans. Industrial Electronics, Vol.40, No.2, pp.259-265 (1993-4)

(35) S. Katsura, K. Irie, and K. Ohishi: "Wideband Force Control by PositionAcceleration Integrated Disturbance Observer", IEEE Trans. Industrial Electronics, Vol.55, No.4, pp.1699-1706 (2008)

Yuki Yokokura (Member) received the B.E. and M.E. degrees in elec-

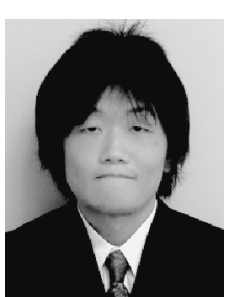
trical engineering from Nagaoka University of Technology, Niigata, Japan, in 2007 and 2009, respectively, and Ph.D. degree in integrated design engineering from Keio University, Yokohama, Japan, in 2011. From 2010 to 2011, he was a JSPS (Japan Society for the Promotion of Science) Research Fellow (DC2 and PD). He was a Visiting Fellow at Keio University, and a Postdoctoral Fellow at Nagaoka University of Technology in 2011. Since 2012, he has been an Assistant Professor with Nagaoka University of Technology. His research interests include motion control, motor drive, powerelectronics, and real-world haptics.

Kiyoshi Ohishi (Senior Member) received the B.S., M.S., and Ph.D.

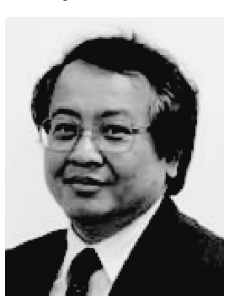
degrees, all in electrical engineering, from Keio University, Yokohama, Japan, in 1981, 1983, and 1986, respectively. From 1986 to 1993, he was an Associate Professor with Osaka Institute of Technology, Osaka, Japan. From 1993 to 2003, he was an Associate Professor with Nagaoka University of Technology, Niigata, Japan. Since August 2003, he has been a Professor at the same university. He is an administration committee member of the IEEE Industrial Electronics Society, the Institute of Electrical Engineers of Japan (IEEJ), the Japan Society of Mechanical Engineers (JSME), the Society of Instrument and Control Engineers (SICE), and the Robotics Society of Japan (RSJ). 